\title{
Symplectic homology and periodic orbits near symplectic submanifolds
}

Kai Cieliebak, Viktor L. Ginzburg and Ely Kerman

\begin{abstract}
We show that a small neighborhood of a closed symplectic submanifold in a geometrically bounded aspherical symplectic manifold has non-vanishing symplectic homology. As a consequence, we establish the existence of contractible closed characteristics on any thickening of the boundary of the neighborhood. When applied to twisted geodesic flows on compact symplectically aspherical manifolds, this implies the existence of contractible periodic orbits for a dense set of low energy values.
\end{abstract}

Mathematics Subject Classification (2000). 53D40, 37J45.

Keywords. Periodic orbits, Hamiltonian flows, symplectic homology, symplectic submanifolds.

\section{Introduction}

The symplectic homology of an open subset of a symplectic manifold is intimately connected with closed characteristics on the shells near the boundary of the subset, $[\mathrm{FH}]$. In this paper we show that for a small neighborhood of a compact symplectic submanifold in a geometrically bounded symplectically aspherical manifold the symplectic homology does not vanish. As a consequence, we establish the existence of contractible closed characteristics on any thickening of the boundary of such a neighborhood.

Symplectic homology was originally introduced and studied in the series of papers [CFH, CFHW, FH, FHW]. Here we follow more recent treatments from [BPS] and, more indirectly, from [Vi]. In particular, we consider the (direct limit) symplectic homology of bounded open sets in a geometrically bounded, symplectically aspherical manifold $(W, \omega)$, and we prove the following:

Theorem 1.1. Let $U$ be a sufficiently small neighborhood of a compact symplectic submanifold $M$ of $W$. Then $\mathrm{SH}^{[a, b)}(U) \neq 0$ for $a<b<0$, when $|a|$ is sufficiently large and $|b|$ is sufficiently small.

This work was partially supported by the NSF and by the faculty research funds of the University of California, Santa Cruz. The third author was fully supported by NSERC. 
This yields the following existence result along the lines of the Weinstein-Moser theorem.

Theorem 1.2. Let $H: W \rightarrow \mathbb{R}$ be a smooth function which attains an isolated minimum on a compact symplectic submanifold $M$ (say, $\left.\left.H\right|_{M}=0\right)$. Then the levels $\{H=\epsilon\}$ carry contractible periodic orbits for a dense set of small values $\epsilon>0$.

As an application we obtain a new existence theorem for twisted geodesic flows.

Corollary 1.3. Let $(M, \sigma)$ be a compact symplectically aspherical manifold and consider the cotangent bundle $T^{*} M$ equipped with the twisted symplectic form $\omega=$ $\omega_{0}+\pi^{*} \sigma$, where $\omega_{0}$ is the standard form on $T^{*} M$ and $\pi: T^{*} M \rightarrow M$ is the natural projection map. Let $H: T^{*} M \rightarrow \mathbb{R}$ be the standard kinetic energy Hamiltonian for a Riemannian metric on $M$, i.e., $H(q, p)=\|p\|^{2}$. Then the Hamiltonian flow of $H$ with respect to $\omega$ has contractible periodic orbits on the levels $\{H=\epsilon\}$ for a dense set of small values $\epsilon>0$.

Theorem 1.2 strengthens or complements some other recent existence results. In particular, it was shown in [GK2], with no assumptions on the symplectic manifold $(W, \omega)$ but when $M$ is a Morse-Bott non-degenerate minimum, that there exists a sequence of energy values $\epsilon_{k} \rightarrow 0$ such that all the levels $\left\{H=\epsilon_{k}\right\}$ carry contractible periodic orbits. Furthermore, under suitable additional conditions, a nontrivial topological lower bound for the number of periodic orbits on every low energy level was established in [GK1, Ke].

For twisted geodesic flows, Corollary 1.3 augments a large family of existence results beyond the applications of the results in [GK1, GK2, Ke] described above. For instance, it was shown in [Mac, Po] that there are contractible periodic orbits on a sequence of energy levels $\left\{H=\epsilon_{k}\right\}$ with $\epsilon_{k} \rightarrow 0$ for any compact manifold $M$ and any closed form $\sigma \neq 0$ with $\left.\sigma\right|_{\pi_{2}(M)}=0$. Also, when $M$ is a torus, there are periodic orbits (not necessarily contractible, e.g., if $\sigma=0$ ) on almost all energy levels for any $\sigma$; see [GK1] and references therein, cf. [Ji]. We refer the reader to the survey [Gi] for a discussion of the results on twisted geodesic flows obtained prior to 1995.

Remark 1.4. In Theorem 1.1, the assumption that $W$ is geometrically bounded can be replaced by other hypotheses that ensure that the symplectic homology of $U$ is well defined. In particular, the theorem also holds for symplectic manifolds with symplectically convex boundary (see Remark 2.3). The choice to consider geometrically bounded manifolds was motivated by our interest in the application to twisted geodesic flows.

The paper is organized as follows. In Section 2, we recall the definition of geometrically bounded manifolds and show that twisted cotangent bundles are geometrically bounded. In Section 3, we briefly review the definitions and properties 
of Floer and symplectic homology which are essential for the proofs of the main theorems. We also show that the non-vanishing property of symplectic homology implies the "nearby" existence of periodic orbits. In Section 4, we show that the symplectic homology in question is non-vanishing and complete the proofs of the main theorems. Finally, in Section 5 we discuss the notion of a relative homological capacity related to these results.

Acknowledgments. The authors are deeply grateful to Başak Gürel and JeanClaude Sikorav for useful discussions and suggestions. The second author would also like to thank the ESI, Vienna for the hospitality during the period when this work was essentially completed. The third author would like to express his gratitude to the Fields Institute and the University of Toronto for their hospitality.

\section{Geometrically bounded symplectic manifolds}

\subsection{Definition of geometrically bounded symplectic manifolds}

In what follows, we will need to use the Floer homology of compactly supported functions on non-compact symplectically aspherical manifolds. In order for this to be well defined, certain conditions on the manifold at infinity must be imposed to ensure that the necessary compactness theorems hold. One standard way to achieve this is to require the symplectic manifold to be in a certain sense convex. However, the manifolds we are most interested in - twisted cotangent bundles do not generally meet this requirement. Hence convexity is replaced here by the less restrictive requirement that the manifold is geometrically bounded. Let us recall the definition.

Definition 2.1. A symplectic manifold $(W, \omega)$ is said to be geometrically bounded if $W$ admits an almost complex structure $J$ and a complete Riemannian metric $g$ such that

GB1. $J$ is uniformly $\omega$-tame, i.e., for some positive constants $c_{1}$ and $c_{2}$ we have

$$
\omega(X, J X) \geq c_{1}\|X\|^{2} \quad \text { and } \quad|\omega(X, Y)| \leq c_{2}\|X\|\|Y\|
$$

for all tangent vectors $X$ and $Y$ to $W$.

GB2. The sectional curvature of $(W, g)$ is bounded from above and the injectivity radius of $(W, g)$ is bounded away from zero.

Observe that if $W$ is compact then condition (GB1) holds automatically when $g, \omega$ and $J$ are compatible, i.e.,

$$
\omega(\cdot, J \cdot)=g(\cdot, \cdot) .
$$

Clearly, for compact manifolds condition (GB2) also holds for any metric, and so every compact symplectic manifold is geometrically bounded. Other examples 
of geometrically bounded manifolds are given in the next section. We refer the reader to Chapters V (by J.-C. Sikorav) and X (by M. Audin, F. Lalonde and L. Polterovich) in [AL] for a more detailed discussion of this concept.

\subsection{Example: twisted cotangent bundles}

Let us recall the definition of a twisted cotangent bundle. Consider a closed manifold $M$ and a closed two-form (magnetic field) $\sigma$ on $M$. Set $W=T^{*} M$. The form $\omega=\omega_{0}+\pi^{*} \sigma$ is called a twisted symplectic form, where $\omega_{0}$ is the standard symplectic form on $T^{*} M$ and $\pi: T^{*} M \rightarrow M$ is the natural projection.

One reason these forms are of interest is because the Hamiltonian flow of the standard kinetic energy Hamiltonian $H: T^{*} M \rightarrow \mathbb{R}$, defined by a Riemannian metric on $M$, describes the motion of a charge on $M$ in the magnetic field $\sigma$. This is also called a twisted geodesic flow.

Proposition 2.2. A twisted cotangent bundle $\left(T^{*} M, \omega\right)$ is geometrically bounded for any closed manifold $M$ and any closed two-form $\sigma$ on $M$.

This proposition is, of course, well known. The fact that $T^{*} M$ satisfies condition (GB2) for the natural metric induced by a metric on $M$, is stated without a proof in $[\mathrm{AL}]$; see p. 96 and p. 286. This assertion is used by Lu in [Lu] to show that a twisted cotangent bundle (again, with a natural metric) is geometrically bounded. For the sake of completeness, we outline a proof of Proposition 2.2 below (for a different choice of metric on $T^{*} M$ ). ${ }^{1}$

Proof. Let $\varphi_{t}$ be the flow on $W=T^{*} M$ formed by fiberwise dilations by the factor $e^{t}$. The standard symplectic form $\omega_{0}$ is homogeneous of degree one with respect to the dilations: $\varphi_{t}^{*} \omega_{0}=e^{t} \omega_{0}$. Pick a fiberwise convex hypersurface $\Sigma$ in $W$, enclosing the zero section $M$. Note that $\Sigma$ has contact type for $\omega_{0}$, but not necessarily for the twisted form $\omega$. Denote by $U$ the closure of the unbounded component of the complement to $\Sigma$ in $W$, i.e., $U=\cup_{t \geq 0} \varphi_{t}(\Sigma)$.

On the vector bundle $\left.T W\right|_{\Sigma}$, pick any fiberwise metric $g$ and a complex structure which are compatible with $\omega_{0}$ in the sense of equation (1). (We also require the radial vectors to be $g$-orthogonal to $\Sigma$.) Let us extend these structures to $U$ so that

$$
\varphi_{t}^{*} g=e^{t} g \text { for } \quad t \geq 0,
$$

i.e., $g$, just as $\omega_{0}$, is homogeneous of degree one with respect to the dilations, and

$$
J \circ\left(\varphi_{t}\right)_{*}=\left(\varphi_{t}\right)_{*} \circ J
$$

Then the metric $g$, the almost complex structure $J$, and $\omega_{0}$ are compatible on $U$ and hence the condition (GB1) holds for these structures. We will show that this

\footnotetext{
1 The authors are grateful to Jean-Claude Sikorav for suggesting to us the idea of this proof.
} 
condition also holds for $g, J$, and $\omega$ for, perhaps, a dilated hypersurface $\Sigma$.

The metric $g$ is obviously complete. Indeed, identifying $U$ with $\Sigma \times[1, \infty)$, we see that the metric $g$ has the form

$$
g=\frac{d t^{2}}{t}+\frac{\left.g\right|_{\Sigma}}{t}
$$

It is then clear that the integral curves $\varphi_{t}(x)$, for $t>0$ and $x \in \Sigma$, are minimizing geodesics of $g$. Thus, the distance from $x$ to $\varphi_{t}(x)$ is equal to $\ln t$ and goes to $\infty$ as $t \rightarrow \infty$. Therefore, every bounded subset of $U$ is contained in some shell $\Sigma \times[1, t]$ and is, hence, relatively compact. This is equivalent to completeness.

It follows readily from (2) that the sectional curvature of $g$ goes to zero as $x \rightarrow \infty$ in $U$. As a consequence, the sectional curvature of $g$ is bounded from above on $U$. Combined with completeness, this implies that the injectivity radius is bounded away from zero. Thus, condition (GB2) holds.

It is also easy to verify condition (GB1). Note that $\pi^{*} \sigma$ is homogeneous of degree zero for $\varphi_{t}$, i.e., $\varphi_{t}^{*} \pi^{*} \sigma=\pi^{*} \sigma$. Then, by a straightforward calculation, it is clear that for any positive constants $c_{1}<1$ and $c_{2}>1$, this condition holds in the smaller set $\varphi_{t}(U)$, provided that $t$ is large enough.

To complete the proof it suffices to extend $g$ and $J$ to the bounded part $W \backslash$ $\varphi_{t}(U)$.

Remark 2.3. Recall that an open symplectic manifold $\left(W, \omega_{0}\right)$ is said to be convex at infinity if there exists: a hypersurface $\Sigma \subset W$ which separates $W$ into one set with compact closure and another, $U$, with non-compact closure; and a flow of symplectic dilations on $U, \varphi_{t}$ (for $t \geq 0$ ), which is transversal to $\partial U=\Sigma$. (See [EG].) An argument similar to the proof above (with the exception of the step dealing with $\pi^{*} \sigma$ ) shows that a symplectic manifold $\left(W, \omega_{0}\right)$ which is convex at infinity is also geometrically bounded. This reasoning together with the fact that $\pi^{*} \sigma$ is "small" compared to $\omega_{0}$ is the main point of the proof above.

\section{Symplectic homology}

\subsection{Floer theory}

In this section we briefly recall the definitions of Floer homology and symplectic homology as well as some of their properties. All the results here are stated without proof. The reader interested in a detailed treatment of Floer homology should consult, for example, [HZ, Sa] or the original sources [Fl1, Fl2, Fl3]. For the definition and properties of symplectic homology the reader is referred to [BPS, CFH, CFHW, FH, FHW, Vi]. ${ }^{2}$

\footnotetext{
2 The definitions of symplectic homology vary considerably from paper to paper. Here we adopt the approach of [BPS].
} 


\subsubsection{Floer homology for negative actions}

Let $(W, \omega)$ be a symplectic manifold, possibly open, which is geometrically bounded (see Definition 2.1). Assume, as well, that $(W, \omega)$ is symplectically aspherical, i.e.,

$$
\left.\omega\right|_{\pi_{2}(W)}=0 \quad \text { and }\left.\quad c_{1}(T W)\right|_{\pi_{2}(W)}=0 .
$$

Denote by $\mathcal{H}$ the space of smooth, compactly supported functions on $S^{1} \times W$ equipped with the strong Whitney $C^{\infty}$-topology, [Hi]. To each $H \in \mathcal{H}$ we can associate the time-dependent Hamiltonian vector field $X_{H}$ which is defined by the equation $d H=-i_{X_{H}} \omega$. The set of contractible periodic orbits of $X_{H}$ with period equal to one is denoted by $\mathcal{P}(H)$.

Let $\mathcal{L} W$ be the space of smooth contractible loops in $W$. We can also associate to each $H \in \mathcal{H}$ the action functional $\mathcal{A}_{H}: \mathcal{L} W \rightarrow \mathbb{R}$ given by

$$
\mathcal{A}_{H}(x)=-\int_{D^{2}} \bar{x}^{*} \omega+\int_{S^{1}} H(t, x) d t
$$

where $\bar{x}: D^{2} \rightarrow W$ is any map which restricts to $S^{1}=\partial D^{2}$ as $x$. This functional is well defined since $\left.\omega\right|_{\pi_{2}(W)}=0$, and the critical points of $\mathcal{A}_{H}$ are exactly the elements of $\mathcal{P}(H)$. The set of critical values of $\mathcal{A}_{H}$ is called the action spectrum and we denote it by

$$
\mathcal{S}(H)=\left\{\mathcal{A}_{H}(x) \mid x \in \mathcal{P}(H)\right\}
$$

Lemma 3.1 ([Sc], [BPS]). The action spectrum $\mathcal{S}(H)$ is compact and nowhere dense for every $H \in \mathcal{H}$. Moreover, it is lower semi-continuous in $H$ in the following sense: for every $H \in \mathcal{H}$ and any neighborhood $V$ of $\mathcal{S}(H)$ in $\mathbb{R}$ there is an open neighborhood $U$ of $H$ such that $\mathcal{S}(\tilde{H}) \subset V$ for all $\tilde{H} \in U$.

Proof. The first statement is proved in [Sc]. The second statement is quoted from [BPS] and can be proven as follows. Let $\psi_{t}^{H}$ be the time- $t$ flow of $X_{H}$. One can associate to $H$ its fixed point set $\operatorname{Fix}(H)=\left\{x \in W \mid \psi_{1}^{H}(x)=x\right\}$ and the function $a_{H}: W \rightarrow \mathbb{R}$ defined by $a_{H}(x)=\mathcal{A}_{H}\left(\psi_{t}^{H}(x)\right)$. Clearly, $a_{H}(\operatorname{Fix}(H))=\mathcal{S}(H)$. The result now follows from the fact that if $\tilde{H} \in \mathcal{H}$ is close to $H$ in the strong Whitney $C^{\infty}$-topology, then $\operatorname{Fix}(\tilde{H})$ is close to $\operatorname{Fix}(H)$ and $a_{\tilde{H}}$ is $C^{1}$-close to $a_{H}$.

In general terms, the Floer homology of $H$ is the homology of the (relative) Morse-Smale-Witten complex of $\mathcal{A}_{H}$ on $\mathcal{L} W$. However, when $W$ is not compact, every point in the complement of supp $H$ is a degenerate 1-periodic orbit (critical point of $\mathcal{A}_{H}$ ) with zero action. To avoid this set, we will only consider the homology generated by the contractible 1-periodic orbits with negative action.

More precisely, for a fixed $a \in(-\infty, 0)$, set

$$
\mathcal{P}^{a}(H)=\left\{x \in \mathcal{P}(H) \mid \mathcal{A}_{H}(x)<a\right\}
$$

and assume that $H$ satisfies the following condition:

$\left(*^{a}\right)$ : Every 1-periodic orbit $x \in \mathcal{P}^{a}(H)$ is nondegenerate. 
Since $\left.c_{1}(T W)\right|_{\pi_{2}(W)}=0$, the elements of $\mathcal{P}^{a}(H)$ are graded by the Conley-Zehnder index $\mu_{\mathrm{CZ}}$; see [Sa]. With this, the Floer complex of $H$ for actions less than $a$ is the graded $\mathbb{Z}_{2}$-vector space

$$
\mathrm{CF}^{a}(H)=\bigoplus_{x \in \mathcal{P}^{a}(H)} \mathbb{Z}_{2} x
$$

To define the Floer boundary operator, we first fix an almost complex structure $J_{g b}$ for which $(W, \omega)$ is geometrically bounded as in Definition 2.1. Let $\mathcal{J}$ be the set of smooth $t$-dependent $\omega$-tame almost complex structures which are $\omega$-compatible near $\operatorname{supp}(H)$ and are equal to $J_{g b}$ outside some compact set. Each $J \in \mathcal{J}$ defines a positive-definite bilinear form on $\mathcal{L} W$. We can then consider the moduli space $\mathcal{M}(x, y, H, J)$ of downward gradient-like trajectories of $\mathcal{A}_{H}$ which go from $x$ to $y$ and have finite energy. For a dense subset, $\mathcal{J}_{\text {reg }}(H) \subset \mathcal{J}$, each moduli space $\mathcal{M}(x, y, H, J)$ is a smooth manifold of dimension $\mu_{\mathrm{CZ}}(x)-\mu_{\mathrm{CZ}}(y)$.

The Floer boundary operator is then defined by

$$
\partial^{H, J} x=\sum_{y \in \mathcal{P}^{a}(H) \text { with } \mu_{\mathrm{CZ}}(x)-\mu_{\mathrm{CZ}}(y)=1} \tau(x, y) y,
$$

where $\tau(x, y)$ stands for the number $(\bmod 2)$ of elements in $\mathcal{M}(x, y, H, J) / \mathbb{R}$ and $\mathbb{R}$ acts (freely) by translation on the gradient-like trajectories. The operator $\partial^{H, J}$ satisfies $\partial^{H, J} \circ \partial^{H, J}=0$ and the resulting Floer homology groups $\operatorname{HF}^{a}(H)$ are independent of the choice of $J \in \mathcal{J}_{\text {reg }}(H)$.

Remark 3.2. Since $(W, \omega)$ with $J_{g b}$ is geometrically bounded and $H$ is compactly supported, there is a uniform $C^{0}$-bound for the elements of $\mathcal{M}(x, y, H, J)$ (see, for example, Chapter V in $[\mathrm{AL}]$ ). Hence, the compactness of the appropriate moduli spaces follows from the usual arguments.

Remark 3.3. In this definition of $\partial^{H, J}$ we ignore matters of orientation by considering only coefficients in $\mathbb{Z}_{2}$.

Remark 3.4. It is unclear whether $\operatorname{HF}^{a}(H)$ depends on the choice of $J_{g b}$. The Floer homology $\mathrm{HF}^{a}(H)$ is independent of this choice if the set of almost complex structures for which $W$ is geometrically bounded is connected.

It will also be useful to consider Floer homology restricted to smaller negative action intervals. More precisely, for constants $-\infty \leq a<b<0$ let $H \in \mathcal{H}^{a, b}$, where

$$
\mathcal{H}^{a, b}=\{H \in \mathcal{H} \mid a, b \notin \mathcal{S}(H)\} .
$$

Assume, as well, that $H$ has property $\left(*^{b}\right)$. Then $\mathrm{CF}^{a}(H)$ is a subcomplex of $\mathrm{CF}^{b}(H)$ and $\operatorname{HF}^{[a, b)}(H)$ is the homology of the quotient complex $\mathrm{CF}^{[a, b)}(H)=$ $\mathrm{CF}^{b}(H) / \mathrm{CF}^{a}(H)$ with the induced boundary operator. 
It follows from Lemma 3.1 that $\mathcal{H}^{a, b}$ is open in $\mathcal{H}$. Moreover, in each component of $\mathcal{H}^{a, b}$, the functions with property $\left(*^{b}\right)$ form a dense set and have locally identical Floer homology groups. Therefore, one can define $\operatorname{HF}^{[a, b)}(H)$ for any $H \in \mathcal{H}^{a, b}$ as the restricted Floer homology of a nearby function in $\mathcal{H}^{a, b}$ with property $\left(*^{b}\right)$.

\subsubsection{Morse-Bott Floer homology}

The extension of the definition of $\operatorname{HF}^{[a, b)}(H)$ to every $H \in \mathcal{H}^{a, b}$ is particularly useful in the Morse-Bott case which we now describe.

A subset $P \subset \mathcal{P}(H)$ is said to be a Morse-Bott manifold of periodic orbits if the set $C_{0}=\{x(0) \mid x \in P\}$ is a compact submanifold of $W$ and $T_{x_{0}} C_{0}=$ $\operatorname{ker}\left(D \phi_{H}^{1}\left(x_{0}\right)-i d\right)$ for every $x_{0} \in C_{0}$. Here $\phi_{H}^{1}$ is the time-1 flow of $X_{H}$.

For such sets of periodic orbits we have the following result which holds for geometrically bounded, symplectically aspherical manifolds.

Theorem 3.5. (Poźniak, [Po, Corollary 3.5.4]; Biran-Polterovich-Salamon, [BPS, Section 5.2].) Let $-\infty \leq a<b<0$ and $H \in \mathcal{H}^{a, b}$. Suppose that the set $P=\left\{x \in \mathcal{P}(H) \mid a<\mathcal{A}_{H}<b\right\}$ is a connected Morse-Bott manifold of periodic orbits. Then $\mathrm{HF}^{[a, b)}(H)$ is isomorphic to $H_{*}\left(P ; \mathbb{Z}_{2}\right)$.

The isomorphism in this theorem does not preserve grading, i.e., $H F_{*}^{[a, b)}(H)=$ $H_{*-j}\left(P ; \mathbb{Z}_{2}\right)$ for some shift $j$ which depends on the linearized flow of $H$ around the orbits in $P$.

\subsubsection{Monotone homotopies}

Let $H, K \in \mathcal{H}$ be two functions with $H(t, x) \geq K(t, x)$ for all $(t, x) \in S^{1} \times W$. Then there exists a monotone homotopy $s \mapsto K_{s}$ from $H$ to $K$, i.e., a smooth family of functions $K_{s} \in \mathcal{H}$ such that

$$
K_{s}= \begin{cases}H & \text { for } s \in(-\infty,-1] \\ K & \text { for } s \in[1, \infty)\end{cases}
$$

and $\partial_{s} K_{s} \leq 0$. If $c<0$ is not a critical value for $\mathcal{A}_{H}$ or $\mathcal{A}_{K}$ and if $H$ and $K$ have nondegeneracy property $\left(*^{c}\right)$, then each monotone homotopy defines a Floer chain map

$$
\sigma_{K H}: \mathrm{CF}^{c}(H) \rightarrow \mathrm{CF}^{c}(K) .
$$

Moreover, every $y \in \mathcal{P}^{c}(K)$ which appears in the image of $x \in \mathcal{P}^{c}(H)$ under $\sigma_{K H}$ satisfies $\mathcal{A}_{K}(y) \leq \mathcal{A}_{H}(x)$. Thus, if $H$ and $K$ are in $\mathcal{H}^{a, b}$ and have property $\left(*^{b}\right)$, then $\sigma_{K H}$ determines a chain map for the quotient complexes

$$
\sigma_{K H}: \mathrm{CF}^{[a, b)}(H) \rightarrow \mathrm{CF}^{[a, b)}(K)
$$

which induces a homomorphism in Floer homology

$$
\sigma_{K H}: \mathrm{HF}^{[a, b)}(H) \rightarrow \mathrm{HF}^{[a, b)}(K) .
$$


Just as the Floer homology groups $\mathrm{HF}^{[a, b)}(H)$ and $\mathrm{HF}^{[a, b)}(K)$ are locally constant on $\mathcal{H}^{a, b}$, so too is the homomorphism $\sigma_{K H}$. This allows one to extend the definition of the homomorphism $\sigma_{K H}$ to functions $H$ and $K$ in $\mathcal{H}^{a, b}$ which may not have the nondegeneracy property $\left(*^{b}\right)$.

The following results concerning these homomorphisms are well known ; see, e.g., [CFH, FH] and [BPS, Sections 4.4 and 4.5].

Lemma 3.6. The homomorphism $\sigma_{K H}$ is independent of the choice of the monotone homotopy $K_{s}$ and satisfies the following identities

$$
\begin{aligned}
\sigma_{K H} \circ \sigma_{H G} & =\sigma_{K G} \text { for } G \geq H \geq K, \\
\sigma_{H H} & =i d \text { for every } H \in \mathcal{H}^{a, b} .
\end{aligned}
$$

Lemma 3.7. If $K_{s}$ is a monotone homotopy from $H$ to $K$ such that $K_{s} \in \mathcal{H}^{a, b}$ for all $s \in[-1,1]$, then $\sigma_{K H}: \mathrm{HF}^{[a, b)}(H) \rightarrow \mathrm{HF}^{[a, b)}(K)$ is an isomorphism.

This last result states that the only way in which the map $\sigma_{K H}$ can fail to be an isomorphism is if periodic orbits, with action equal to $a$ or $b$, are created during the homotopy. In fact, this is a particular instance of the following more general phenomenon.

Let $K_{s}$ be a monotone homotopy from $H$ to $K$, as above. Assume, for some $c<a$, that $K \in \mathcal{H}^{c, b}$ and

$$
\sigma_{K H}\left(\mathrm{CF}^{a}(H)\right) \subset \mathrm{CF}^{c}(K) .
$$

Then $\sigma_{K H}$ induces a homomorphism $\hat{\sigma}_{K H}$ from $\operatorname{HF}^{[a, b)}(H)$ to $\operatorname{HF}^{[c, b)}(K)$. (We use the hat " ^" to emphasize the fact that the map goes between Floer homology groups restricted to different intervals of actions.) In this case, we get the following generalization of Lemma 3.7.

Lemma 3.8. For $s \in[-1,1]$, let $a_{s}$ be a continuous family of numbers, less than $b$, such that $a_{-1}=a$ and $a_{1}=c$. If the monotone homotopy $K_{s}$ satisfies $K_{s} \in \mathcal{H}^{a_{s}, b}$ for every $s \in[-1,1]$, then

$$
\hat{\sigma}_{K H}: \operatorname{HF}^{[a, b)}(H) \rightarrow \mathrm{HF}^{[c, b)}(K)
$$

is an isomorphism.

Remark 3.9. To define the chain maps above and to prove that the homomorphisms $\sigma_{K H}$ and $\hat{\sigma}_{K H}$ are independent of the choice of the monotone homotopy (as long as $\left.K_{s} \in \mathcal{H}^{a_{s}, b}\right)$, one needs to consider moduli spaces which are defined using generic parameterized families in $\mathcal{J}$. Each such family is constant and equal to $J_{g b}$ outside a compact set in $W$. Hence, as in Remark 3.2, the necessary compactness statements follow from the standard arguments. 


\subsection{Symplectic homology}

\subsubsection{Direct limits and exhausting sequences}

Following closely the discussion in [BPS, Sections 4.6 and 4.7], we recall here the algebraic constructions needed to define symplectic homology. Let $(I, \preceq)$ be a partially ordered set which is thought of as a category with precisely one morphism from $i$ to $j$ whenever $i \preceq j$. A partially ordered system of $R$-modules over $I$ is a functor from this category to the category of $R$-modules. This is written as a pair $(G, \sigma)$ where $G$ assigns to each $i \in I$ an $R$-module $G_{i}$ and $\sigma$ assigns to each $i \preceq j$ an $R$-module homomorphism $\sigma_{j i}: G_{i} \rightarrow G_{j}$ such that $\sigma_{k j} \circ \sigma_{j i}=\sigma_{k i}$ for $i \preceq j \preceq k$ and $\sigma_{i i}$ is the identity on $G_{i}$.

The partially ordered set $(I, \preceq)$ is said to be upwardly directed if for each $i, j \in I$ there is an $l \in I$ such that $i \preceq l$ and $j \preceq l$. In this case, the functor $(G, \sigma)$ is called a directed system of $R$-modules and its direct limit is defined as

$$
\stackrel{\lim }{\longrightarrow}=\left\{(i, x) \mid i \in I, x \in G_{i}\right\} / \sim,
$$

where $(i, x) \sim(j, y)$ if and only if there exists an $l \in I$ such that $i \preceq l, j \preceq l$ and $\sigma_{l i}(x)=\sigma_{l j}(y)$.

Note also that for each $i \in I$ there is a natural homomorphism

$$
\iota_{i}: G_{i} \rightarrow \underset{\lim }{\longrightarrow}
$$

which takes $x$ to the equivalence class $[i, x]$.

It is possible to actually compute a direct limit given an exhausting sequence. This is a sequence $\left\{i_{\nu}\right\}_{\nu \in \mathbb{Z}^{+}} \subset I$ with the following two properties:

- For every $\nu \in \mathbb{Z}^{+}$we have $i_{\nu} \preceq i_{\nu+1}$ and $\sigma_{i_{\nu+1} i_{\nu}}: G_{i_{\nu}} \rightarrow G_{i_{\nu+1}}$ is an isomorphism.

- For every $i \in I$ there exists a $\nu \in \mathbb{Z}^{+}$such that $i \preceq i_{\nu}$.

Lemma 3.10. For such a sequence the map

$$
\iota_{i_{\nu}}: G_{i_{\nu}} \rightarrow \underset{\lim }{\longrightarrow} G
$$

is an isomorphism for all $\nu \in \mathbb{Z}^{+}$.

\subsubsection{Construction of $\mathrm{SH}^{[a, b)}(U)$}

Let $U \subset W$ be a bounded open subset. We are now in a position to define an invariant $\mathrm{SH}^{[a, b)}(U)$ of $U$, called the symplectic homology of $U$ for the action interval $[a, b)$. This is done by taking the direct limit of certain Floer homology groups.

Let

$$
\mathcal{H}^{a, b}(U)=\left\{H \in \mathcal{H}^{a, b}\left|\operatorname{supp}(H) \subset S^{1} \times U, H\right|_{S^{1} \times U} \leq 0\right\} .
$$

This set has a partial order given by

$$
H \preceq K \Longleftrightarrow H \geq K,
$$


and it is clearly upwardly directed. Note that $\mathcal{H}^{a, b}(U) \neq \emptyset$ for any $-\infty \leq a<b<0$.

To each $H \in \mathcal{H}^{a, b}(U)$, we can associate the $\mathbb{Z}_{2}$-vector space given by the Floer homology groups $\mathrm{HF}^{[a, b)}(H)$. Furthermore, for $H \preceq K$ we have, by Lemma 3.6, a homomorphism $\sigma_{K H}$ which satisfies the required identities to make this a directed system. Passing to the direct limit, we set

$$
\mathrm{SH}^{[a, b)}(U)=\underline{\lim } \operatorname{HF}^{[a, b)}(H) .
$$

As, above, for each $H \in \mathcal{H}^{a, b}(U)$ we have the natural homomorphism

$$
\iota_{H}: \mathrm{HF}^{[a, b)}(H) \rightarrow \mathrm{SH}^{[a, b)}(U) .
$$

Remark 3.11. The condition that $\operatorname{supp}(H) \subset S^{1} \times U$ is sometimes replaced by the weaker requirement $\operatorname{supp}(H) \subset S^{1} \times \bar{U}$, see $[\mathrm{CFH}, \mathrm{FH}]$, where the bar denotes the closure. This results in a symplectic homology with properties similar to the ones considered here and in [BPS]. However, it is likely that the different definitions can yield different symplectic homology groups when $\partial U$ fails to have contact type. It is also worth noticing that the symplectic homology defined with $\operatorname{supp}(H) \subset S^{1} \times \bar{U}$ cannot be calculated by passing to exhausting sequences (but rather exhausting families of functions). For example, the functions $H_{\nu}$ from Section 3.3 could not be used to calculate such symplectic homology.

We now recall the definitions and properties of some of the natural maps introduced in $[\mathrm{FH}]$ for symplectic homology. The reader interested in further details and the proofs of these properties should consult [BPS, CFH, FH].

\subsubsection{Monotonicity maps}

Let $V \subset U$ also be a bounded open subset of $W$. Then we have the natural inclusion

$$
\mathcal{H}^{a, b}(V) \subset \mathcal{H}^{a, b}(U)
$$

and the induced natural homomorphism in symplectic homology

$$
\phi_{U V}: \mathrm{SH}^{[a, b)}(V) \rightarrow \mathrm{SH}^{[a, b)}(U),
$$

which is called a monotonicity map.

Lemma 3.12. For a nested sequence $U_{1} \subset U_{2} \subset U_{3}$ of bounded open subsets of $W, \phi_{U_{3} U_{2}} \circ \phi_{U_{2} U_{1}}=\phi_{U_{3} U_{1}}$.

The relationship of the monotonicity map and the map $\iota_{H}$ is described, for 
$H \in \mathcal{H}^{a, b}(V)$, by the following commutative diagram

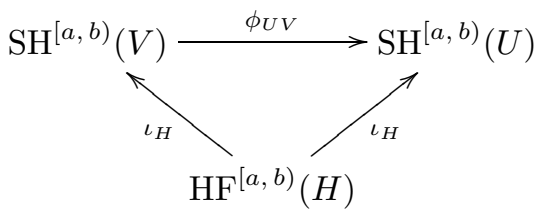

\subsubsection{Exact triangles}

For $-\infty \leq a \leq b \leq c<0$ and a function $H$ we have the short exact sequence of complexes

$$
0 \rightarrow \mathrm{CF}^{[a, b)}(H) \rightarrow \mathrm{CF}^{[a, c)}(H) \rightarrow \mathrm{CF}^{[b, c)}(H) \rightarrow 0
$$

If $a, b, c \notin \mathcal{S}(H)$, this sequence yields the exact homology triangle $\triangle_{a, b, c}(H)$ given by

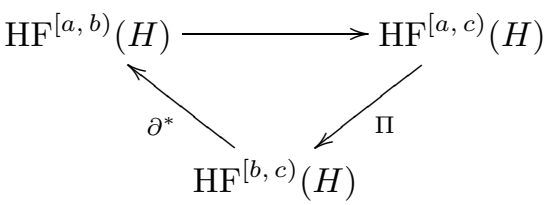

Passing to the direct limit, we obtain an exact triangle $\triangle_{a, b, c}(U)$ for the symplectic homology for $U$ :

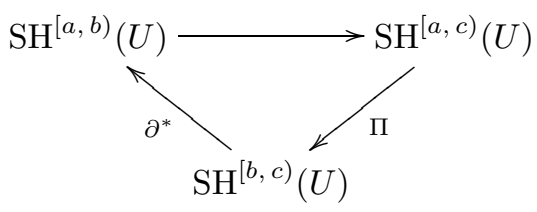

Note that the map $\partial^{*}$ has degree -1 in both diagrams.

Finally, let us describe a relation between the exact triangles and the homomorphisms induced by monotone homotopies which will be crucial to us later on. Let $\left\{K_{s}\right\}$ be a monotone homotopy between functions $H, K \in \mathcal{H}^{a, b}(U)$ with $H \geq K$. As mentioned above, this induces a homomorphism

$$
\sigma_{K H}: \mathrm{HF}^{[a, b)}(H) \rightarrow \mathrm{HF}^{[a, b)}(K) .
$$

If, for some $c<a$, we have $K \in \mathcal{H}^{c, b}$ and $\sigma_{K H}\left(\mathrm{CF}^{a}(H)\right) \subset \mathrm{CF}^{c}(K)$, then $K_{s}$ also induces a homomorphism

$$
\hat{\sigma}_{K H}: \operatorname{HF}^{[a, b)}(H) \rightarrow \mathrm{HF}^{[c, b)}(K) .
$$


Lemma 3.13. The following diagram commutes

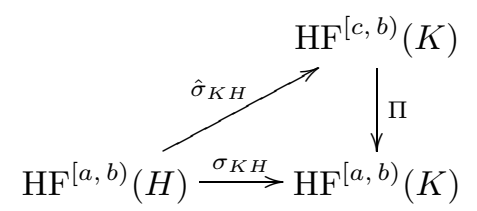

where $\Pi$ is the map from the exact triangle $\triangle_{c, a, b}(K)$.

Proof. Using the explicit definition of the maps $\sigma_{K H}$ and $\hat{\sigma}_{K H}$ (see $[\mathrm{CFH}, \mathrm{FH}]$ ), it is not hard to see that the diagram commutes even on the chain level.

\subsection{Symplectic homology and "nearby existence" of periodic orbits}

Let $\Sigma$ be a smooth hypersurface in $(W, \omega)$. The kernel of the restriction of $\omega$ to $\Sigma$ is 1-dimensional everywhere. The corresponding foliation of $\Sigma$ is called the characteristic foliation and its leaves are called characteristics. If $\Sigma$ is a regular level set for a smooth function $H: W \rightarrow \mathbb{R}$, then the Hamiltonian vector field $X_{H}$ is tangent to the characteristic foliation and so closed characteristics on $\Sigma$ correspond to periodic orbits of $X_{H}$ on $\Sigma$.

If $U$ is a bounded open subset of $W$ with smooth boundary $\partial U$, then the symplectic homology $\mathrm{SH}^{[a, b)}(U)$ detects the contractible closed characteristics on or near $\partial U$. To make this idea more precise, set $\Sigma=\partial U$ and define a thickening of $\Sigma$ to be a map $\Psi:[-1,0] \times \Sigma \rightarrow W$ which is a diffeomorphism on its image and satisfies $\Psi(\{0\} \times \Sigma)=\Sigma$ and $\Psi([-1,0) \times \Sigma) \subset U$. The following result, in a slightly different form, goes back to $[\mathrm{FH}]$.

Proposition 3.14. Suppose that $\mathrm{SH}^{[a, b)}(U) \neq 0$ for $-\infty<a \leq b \leq 0$. Then for any thickening $\Psi$ of $\Sigma=\partial U$ there is a sequence $\left\{t_{i}\right\} \subset[-1,0)$, converging to 0 , such that all corresponding hypersurfaces $\Sigma_{t_{i}}=\Psi\left(t_{i} \times \Sigma\right)$ carry contractible closed characteristics.

Proof. Assume that no such sequence exists. Then there exists a number $\lambda \in$ $[-1,0)$ such that none of the hypersurfaces $\Sigma_{t}$ for $t \in[\lambda, 0)$ contains a contractible closed characteristic. Consider a sequence of smooth functions $f_{\nu}:[\lambda, 0] \rightarrow(-\infty, 0]$ with the following properties:

- $f_{\nu}(t)= \begin{cases}0 & \text { for } t \in[\lambda / 4 \nu, 0], \\ a-\nu & \text { for } t \in[\lambda, \lambda(1-1 / 4 \nu)] .\end{cases}$

- $f_{\nu}^{\prime}(t)>0$ for $t \in(\lambda(1-1 / 4 \nu), \lambda / 4 \nu)$.

- $f_{\nu}(t) \geq f_{\nu+1}(t)$ for all $\nu \in \mathbb{Z}^{+}$and $t \in[\lambda, 0]$. 
Using these functions, we construct a sequence of Hamiltonians $H_{\nu}: W \rightarrow \mathbb{R}$ by setting

$$
H_{\nu}(x)= \begin{cases}0 & \text { for } x \in W \backslash \bar{U}, \\ f_{\nu}(t) & \text { for } x \in \Sigma_{t}, t \in[-1,0), \\ a-\nu & \text { for } x \in U \backslash \Psi([-1,0) \times \Sigma) .\end{cases}
$$

The functions $\left\{H_{\nu}\right\}$ clearly have the second property of an exhausting sequence and satisfy $H_{\nu} \geq H_{\nu+1}$. We claim that the maps $\sigma_{H_{\nu+1} H_{\nu}}$ are (trivially) isomorphisms and the functions $\left\{H_{\nu}\right\}$ indeed form an exhausting sequence. By construction, the Hamiltonian vector fields $X_{H_{\nu}}$ are nonzero only on the level sets corresponding to the hypersurfaces $\left\{\Sigma_{t}\right\}_{t \in[\lambda, 0)}$. On these level sets, the trajectories of the $X_{H_{\nu}}$ correspond to characteristics and, by assumption, none of these can be closed and contractible. Away from the level sets $\left\{\Sigma_{t}\right\}_{t \in[\lambda, 0)}$, the trajectories are all constant and have action either equal to zero or less than $a$. Thus, the Floer homology groups $\mathrm{HF}^{[a, b)}\left(H_{\nu}\right)$ are trivial for all $\nu$ and the functions $H_{\nu}$ form an exhausting sequence. This implies that

$$
\mathrm{SH}^{[a, b)}(U)=\iota_{H_{\nu}}\left(\mathrm{HF}^{[a, b)}\left(H_{\nu}\right)\right)=0,
$$

which is a contradiction.

\section{Proofs of the main theorems}

We are now in a position to prove Theorem 1.1 and Theorem 1.2. Since most of this section is devoted to the proof of the first of these theorems, we recall its assertion for the sake of convenience.

Theorem 4.1. Let $M$ be a compact symplectic submanifold of a geometrically bounded symplectically aspherical manifold $(W, \omega)$. Let $U$ be a sufficiently small neighborhood of $M$ in $W$. Then $\mathrm{SH}^{[a, b)}(U) \neq 0$ for $a<b<0$, when $|a|$ is sufficiently large and $|b|$ is sufficiently small.

Combining Theorem 4.1 and Proposition 3.14, we obtain Theorem 1.2 as follows. The symplectic submanifold $M$ is assumed to be an isolated local minimum of $H: W \rightarrow \mathbb{R}$ with $\left.H\right|_{M}=0$. Hence, all level sets $\{H=\epsilon\}$ have connected components which are close to $M$ and bound small neighborhoods of $M$, when $\epsilon>0$ is small. We denote these neighborhoods by $V_{\epsilon}$, i.e., $V_{\epsilon}$ is connected component of the sub-level set $\{H<\epsilon\}$ which contains $M$.

Let $\epsilon_{0}$ be a regular value of $H$ which is small enough for Theorem 4.1 to apply to the neighborhood $V_{\epsilon_{0}}$ of $M$. By Proposition 3.14 there is a sequence of level sets converging to the connected component of $\left\{H=\epsilon_{0}\right\}$ bounding $V_{\epsilon_{0}}$, all of which contain contractible closed characteristics and hence contractible periodic orbits of $X_{H}$. The same argument can now be applied to any regular value $\epsilon<\epsilon_{0}$ and 
the existence of contractible periodic orbits on a dense set of small level sets then follows from the fact that regular values are dense.

\subsection{Proof of Theorem 4.1}

Let $E \rightarrow M$ be the symplectic normal bundle to $M$, i.e., the symplectic orthogonal complement to $T M$ in $\left.T W\right|_{M}$. We first recall that a neighborhood of the zero section in $E$ has a natural symplectic structure. Moreover, on this neighborhood, there exists a fiberwise quadratic Hamiltonian whose flow is periodic. This can be seen as follows.

Let us equip $E$ with a Hermitian metric which is compatible with the fiberwise symplectic structure on $E$. We denote by $\rho: E \rightarrow \mathbb{R}$ the square of the fiberwise norm, i.e., $\rho(z)=\|z\|^{2}$. Recall that $E$ has a canonical fiberwise one-form whose differential is the fiberwise symplectic form. (The value of this form at $z \in E$ is equal to the contraction of the fiberwise symplectic form by $z$.)

Fixing a Hermitian connection on $E$, we extend this fiberwise one-form to a genuine one-form $\theta$ on $E$. Then the form

$$
\omega_{E}=\frac{1}{2} d \theta+\sigma
$$

is symplectic on a neighborhood of the zero section in $E$. Here we have identified $\sigma=\left.\omega\right|_{M}$ with its pull-back to $E$.

It is not hard to see that all orbits of the Hamiltonian flow of the function $\rho: E \rightarrow \mathbb{R}$ are periodic with period $\pi$, just as for the square of the standard norm on $\mathbb{R}^{2 n}$. (In fact, $i_{X} \omega_{E}=z / 2$ everywhere on $E$, where $X$ is the vector field generating the standard fiberwise Hopf action.) This fact will be essential for the calculation of the Floer homology of a small tubular neighborhood of $M$ in $W$.

By the symplectic neighborhood theorem, a sufficiently small neighborhood of $M$ in $(W, \omega)$ is symplectomorphic to a small neighborhood of $M$ in $\left(E, \omega_{E}\right)$. From now on, we will assume that this identification has been made. Hence, in what follows, $\omega=\omega_{E}$ and $\rho$ is regarded as a function on a neighborhood of $M$ in $W$. Sometimes, we will write $\rho(z)$ as $\|z\|^{2}$.

Denote by $B_{r}$ the disc bundle of radius $r$ in $E$. The key to the proof of Theorem 4.1 is the following result.

Proposition 4.2. Let $0<r<R$ be sufficiently small and assume that

$$
a \in\left[-\infty,-\pi R^{2}\right) \quad \text { and } \quad b \in\left[-\pi r^{2}, 0\right) .
$$

Then

$$
\mathrm{SH}_{n_{0}}^{[a, b)}\left(B_{r}\right)=\mathrm{SH}_{n_{0}}^{[a, b)}\left(B_{R}\right)=\mathbb{Z}_{2},
$$

where

$$
n_{0}=\frac{1}{2}(\operatorname{dim} M-\operatorname{codim} M),
$$


and the monotonicity map $\phi_{B_{R} B_{r}}: \mathrm{SH}_{n_{0}}^{[a, b)}\left(B_{r}\right) \rightarrow \mathrm{SH}_{n_{0}}^{[a, b)}\left(B_{R}\right)$ is an isomorphism.

Remark 4.3. In [FHW], Floer, Hofer and Wysocki compute the symplectic homology of ellipsoids in $\mathbb{R}^{2 n}$ and prove results which are analogous to Proposition 4.2 for $E=\mathbb{R}^{2 n}$ (see Corollary 2 and Proposition 6). Our proof of Proposition 4.2 is similar in spirit to that in [FHW]. However, instead of using explicitly perturbed non-degenerate Hamiltonians as in [FHW], we use Poźniak's theorem (see Theorem 3.5) to calculate the relevant Floer homology groups. This is motivated by the successful use of this technique in [BPS].

Remark 4.4. The hypothesis that $R>0$ is small is only needed to guarantee that $B_{R}$ is contained in $W$ for which the Floer homology is defined. This hypothesis can sometimes be relaxed. For example, if $\omega_{E}$ is symplectic on the entire total space of $E$ and $(E, \omega)$ is geometrically bounded, we can take arbitrarily large radii $R$ and $r$.

Theorem 4.1 follows immediately from Proposition 4.2. Indeed, when $U$ is small enough, we may assume that $U \subset B_{R}$ and, since $M \subset U$, the disc bundle $B_{r}$ is contained in $U$ for sufficiently small $r>0$, i.e.,

$$
B_{r} \subset U \subset B_{R} \text {. }
$$

By Lemma 3.12, the inclusion map $\phi_{B_{R} B_{r}}$ factors as

$$
\mathrm{SH}^{[a, b)}\left(B_{r}\right) \rightarrow \mathrm{SH}^{[a, b)}(U) \rightarrow \mathrm{SH}^{[a, b)}\left(B_{R}\right),
$$

and by Proposition 4.2, the map $\phi_{B_{R} B_{r}}$ is a nontrivial isomorphism for suitable negative constants $a$ and $b$. Thus, we conclude that $\mathrm{SH}^{[a, b)}(U) \neq 0$.

\subsection{Proof of Proposition 4.2}

\subsubsection{Outline of the proof}

First we consider the following diagram which allows us to work in the setting of Floer homology:

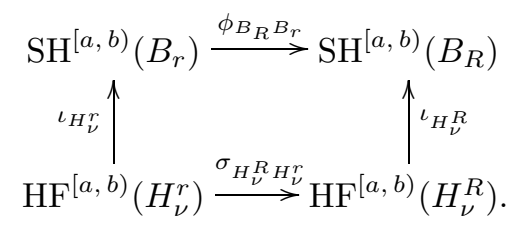

Here, $\left\{H_{\nu}^{r}\right\}_{\nu \in \mathbb{Z}^{+}}$and $\left\{H_{\nu}^{R}\right\}_{\nu \in \mathbb{Z}^{+}}$are chosen to be upward exhausting sequences for the symplectic homology groups $\mathrm{SH}^{[a, b)}\left(B_{r}\right)$ and $\mathrm{SH}^{[a, b)}\left(B_{R}\right)$, respectively. Hence, the two vertical arrows are isomorphisms (for sufficiently large $\nu$ ). For large enough $\nu$, the functions $H_{\nu}^{r}$ and $H_{\nu}^{R}$ belong to $\mathcal{H}^{a, b}$ and $H_{\nu}^{r} \geq H_{\nu}^{R}$. Hence, 
the map $\sigma_{H_{\nu}^{R} H_{\nu}^{r}}$ is well defined. The fact that the diagram commutes then follows easily from diagram (3) and the definition of the direct limit.

By diagram (4), Proposition 4.2 will follow from the two results below which concern the restricted Floer homology of the functions $H_{\nu}^{s}$. In particular, these results imply that the groups $\operatorname{HF}_{n_{0}}^{[a, b)}\left(H_{\nu}^{r}\right)$ and $\operatorname{HF}_{n_{0}}^{[a, b)}\left(H_{\nu}^{R}\right)$ are nontrivial, and that $\sigma_{H_{\nu}^{R} H_{\nu}^{r}}$ is an isomorphism between them.

Claim 4.5. Let $n_{0}=\frac{1}{2}(\operatorname{dim} M-\operatorname{codim} M)$. For every $s \in[r, R]$ and any $c<a$ which is not a negative integer multiple of $\pi s^{2}$, the map

$$
\Pi: \operatorname{HF}_{n_{0}}^{[c, b)}\left(H_{\nu}^{s}\right) \rightarrow \operatorname{HF}_{n_{0}}^{[a, b)}\left(H_{\nu}^{s}\right)
$$

is (eventually) an isomorphism and

$$
\mathrm{HF}_{n_{0}}^{[c, b)}\left(H_{\nu}^{s}\right)=\operatorname{HF}_{n_{0}}^{[a, b)}\left(H_{\nu}^{s}\right)=\mathbb{Z}_{2} .
$$

Here, $\Pi$ is the map from the exact triangle $\triangle_{c, a, b}\left(H_{\nu}^{s}\right)$.

Claim 4.6. Let $k^{\prime}$ be the largest integer in $\left(0,-a / \pi r^{2}\right)$ and let $s_{0}, s_{1} \in[r, R]$ satisfy

$$
s_{0}<s_{1}<\sqrt{1+1 /\left(k^{\prime}+1\right)} s_{0} .
$$

Then there is a constant $c$, satisfying $c<a$, such that the map

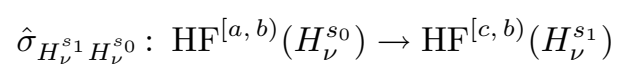

is (eventually) well defined and an isomorphism. Moreover, $c$ can be chosen not to be a negative integer multiple of $\pi s_{1}^{2}$.

Claim 4.5 implies that the groups $\operatorname{HF}_{n_{0}}^{[a, b)}\left(H_{\nu}^{r}\right)$ and $\operatorname{HF}_{n_{0}}^{[a, b)}\left(H_{\nu}^{R}\right)$ are both isomorphic to $\mathbb{Z}_{2}$. Then, it just remains to show that

$$
\sigma_{H_{\nu}^{R} H_{\nu}^{r}}: \operatorname{HF}_{n_{0}}^{[a, b)}\left(H_{\nu}^{r}\right) \rightarrow \operatorname{HF}_{n_{0}}^{[a, b)}\left(H_{\nu}^{R}\right)
$$

is an isomorphism. To see this, let $s_{0}$ and $s_{1}$ satisfy the assumptions of Claim 4.6 and consider the following version of the commutative diagram from Lemma 3.13:

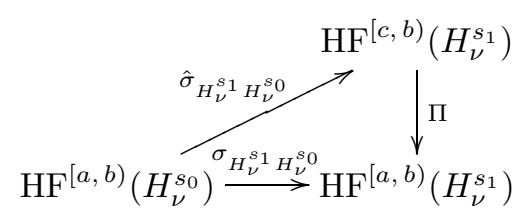

Together, Claims 4.5 and 4.6 imply that the map

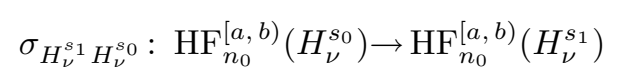

is an isomorphism. Now, by Lemma 3.6, for any sequence $\left\{s_{i}\right\}_{i=1, \ldots, k}$ with

$$
r=s_{0}<s_{1}<\cdots<s_{k-1}<s_{k}=R,
$$


we can decompose $\sigma_{H_{\nu}^{R} H_{\nu}^{r}}$ as

$$
\sigma_{H_{\nu}^{R} H_{\nu}^{r}}=\sigma_{H_{\nu}^{R} H_{\nu}^{s_{k-1}}} \circ \sigma_{H_{\nu}^{s_{k-1}} H_{\nu}^{s_{k-2}}} \circ \cdots \circ \sigma_{H_{\nu}^{s_{1}} H_{\nu}^{s_{r}}} .
$$

Choosing the sequence $s_{1}, \ldots, s_{k}$ so that $s_{i}<s_{i+1}<\sqrt{1+1 /\left(k^{\prime}+1\right)} s_{i}$, we see that $\sigma_{H_{\nu}^{R} H_{\nu}^{r}}$ is an isomorphism from $\operatorname{HF}_{n_{0}}^{[a, b)}\left(H_{\nu}^{r}\right)$ to $\operatorname{HF}_{n_{0}}^{[a, b)}\left(H_{\nu}^{R}\right)$.

\subsubsection{Rescaling}

For simplicity, we will assume that $r=1$ and $R>1$. This can always be achieved by scaling the symplectic structure on $W$ and the metric on $E$. In this case, the fixed constants $a$ and $b$ satisfy $a<-\pi R^{2}<-\pi<b<0$, and $k^{\prime}$ is the largest integer in $(0,-a / \pi)$.

\subsubsection{The functions $H_{\nu}^{s}$}

Here, we construct the functions $H_{\nu}^{s}$, describe their closed orbits with period equal to one, and prove that for each $s \in[1, R]$ the sequence $\left\{H_{\nu}^{s}\right\}$ is (eventually) upwardly exhausting for $\mathrm{SH}^{[a, b)}\left(B_{s}\right)$.

We begin by constructing the exhausting sequence $\left\{H_{\nu}\right\} \equiv\left\{H_{\nu}^{1}\right\}$ for $\mathrm{SH}^{[a, b)}\left(B_{1}\right)$. These functions depend only on the norm of the fibre variable, i.e., $H_{\nu}=H_{\nu}(\|z\|)$. The functions $H_{\nu}^{s}$ are then obtained by rescaling the argument as $H_{\nu}^{s}(\|z\|)=$ $H_{\nu}(\|z\| / s)$.

Consider a sequence of smooth nondecreasing functions $f_{\nu}:[0, \infty) \rightarrow(-\infty, 0]$, for $\nu \in \mathbb{Z}^{+}$, with the following properties (see Figure 1):

(F1) $f_{\nu}(t)= \begin{cases}0 & \text { for } t \in\left[1-1 / 2^{\nu+2}, \infty\right), \\ f_{\nu}(0) & \text { for } t \in\left[0,1-1 / 2^{\nu}\right] .\end{cases}$

(F2) $f_{\nu}(t) \in\left[-1 / 2^{\nu+2}, 0\right]$ for $t \in\left(1-1 / 2^{\nu+1}, 1-1 / 2^{\nu+2}\right)$.

(F3) $f_{\nu}^{\prime}(t)=2^{2 \nu+2}$ for all $t \in\left[1-3 / 2^{\nu+2}, 1-1 / 2^{\nu+1}\right]$.

(F4) $f_{\nu}^{\prime \prime}>0$ for $t \in\left(1-1 / 2^{\nu}, 1-3 / 2^{\nu+2}\right)$ and $f_{\nu}^{\prime \prime}<0$ for $t \in\left(1-1 / 2^{\nu+1}, 1-1 / 2^{\nu+2}\right)$.

On $B=B_{1}$, set

$$
H_{\nu}(z)=f_{\nu}\left(\|z\|^{2}\right)
$$

and extend this function to be identically zero on $W \backslash B$.

Recall that on the neighborhood of $M$ where $\omega_{E}$ is symplectic, the Hamiltonian flow of $\rho(z)=\|z\|^{2}$ is totally periodic with period $\pi$. Hence, for each $\nu$, the flow of $X_{H_{\nu}}$ is totally periodic and the trajectories on each level set of $H_{\nu}$ all have the same period. The level sets which consist of non-constant closed orbits with period equal to one correspond to the solutions of the equations

$$
f_{\nu}^{\prime}\left(\|z\|^{2}\right)=k \pi
$$




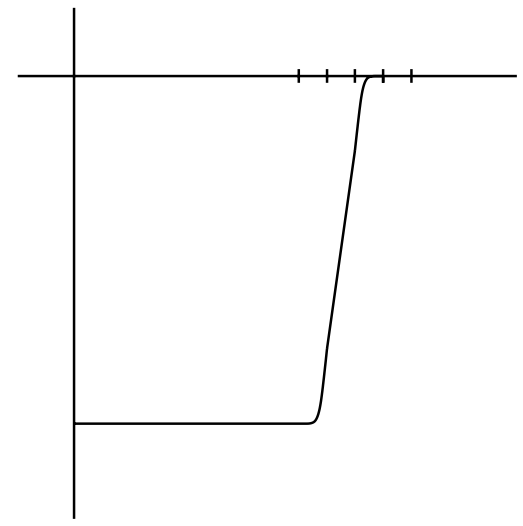

FIG. 1. The functions $f_{\nu}$

where $k$ is a positive integer. These solutions are easy to classify. When $k$ is in the interval $\left[1,2^{\nu+3} / \pi\right)$, there are exactly two levels of $H_{\nu}$ where equation (6) holds:

$$
\begin{aligned}
& \|z\|^{2}=t_{\nu, k}^{-}, \quad \text { where } t_{\nu, k}^{-} \in\left[1-1 / 2^{\nu}, 1-3 / 2^{\nu+2}\right), \text { and } \\
& \|z\|^{2}=t_{\nu, k}^{+}, \quad \text { where } t_{\nu, k}^{+} \in\left(1-1 / 2^{\nu+1}, 1-1 / 2^{\nu+2}\right] .
\end{aligned}
$$

Since $2^{2 \nu+2} \notin \pi \mathbb{Z}^{+}$, there are no other solutions to (6).

Remark 4.7. As $\nu$ increases, one just obtains new solutions $t_{\nu, k}^{ \pm}$of equation (6) for increasingly large values of $k$.

We now show that, for large enough $\nu$, only the orbits on the levels $\|z\|^{2}=$ $t_{\nu, k}^{+} \in\left(1-1 / 2^{\nu+1}, 1-1 / 2^{\nu+2}\right)$ with $k \in[1,-a / \pi)$ have action in $(a, b]$. By a simple calculation, the action of each of the orbits on the level set $\|z\|^{2}=t_{\nu, k}^{ \pm}$is equal to

$$
\mathcal{A}_{\nu, k}^{ \pm}(1)=f_{\nu}\left(t_{\nu, k}^{ \pm}\right)-t_{\nu, k}^{ \pm} \pi k
$$

Since $f_{\nu}(t)<-2^{\nu}$ for all $t \in\left[0,1-3 / 2^{\nu+2}\right]$, the actions $\mathcal{A}_{\nu, k}^{-}$decrease exponentially to negative infinity as $\nu \rightarrow \infty$. Hence, the orbits on the levels $\|z\|^{2}=t_{\nu, k}^{-}$will eventually have action less than $a$. It is also easy to check that the actions $\mathcal{A}_{\nu, k}^{+}(1)$ decrease monotonically to $-\pi k$ as $\nu \rightarrow \infty$. Thus, because $b>-\pi$, all the levels $\|z\|^{2}=t_{\nu, k}^{+}$will eventually have action less than $b$. Similarly, the levels $\|z\|^{2}=t_{\nu, k}^{+}$, with $k \in\left(-a / \pi, 2^{\nu+3} / \pi\right)$, will eventually have action less than $a$.

From this discussion we also see that the functions $\left\{H_{\nu}\right\}$ belong to $\mathcal{H}^{a, b}$ for all sufficiently large $\nu$.

Lemma 4.8. The sequence $\left\{H_{\nu}\right\}$ is (eventually) upwardly exhausting for the symplectic homology $\mathrm{SH}^{[a, b)}(B)$. 
Proof. The only property that is not immediately obvious is that $\sigma_{H_{\nu+1} H_{\nu}}$ is an isomorphism for all sufficiently large $\nu$. To see this, consider $\nu$ as a continuous parameter and look at the monotone homotopy $\left\{H_{\nu+\tau}\right\}_{\tau \in[0,1]}$ between $H_{\nu}$ and $H_{\nu+1}$. When $\nu$ is large enough, the new periodic orbits with period one which appear as $\tau$ goes from zero to one will all have action less than $a$. (This follows from Remark 4.7 and the fact that $\mathcal{A}_{\nu, k}^{+}(1) \rightarrow-k \pi$ as $\nu \rightarrow \infty$.) Hence, $\left\{H_{\nu+\tau}\right\} \in \mathcal{H}^{a, b}$ for all $\tau \in[0,1]$ and Lemma 4.8 immediately follows from Lemma 3.7.

Remark 4.9. The same argument shows that $\left\{H_{\nu}\right\}$ is (eventually) upwardly exhausting for every $\mathrm{SH}^{[c, d)}(B)$ with $a \leq c \leq d<0$.

For $1 \leq s \leq R$, we now define the functions $H_{\nu}^{s}$ by the equation

$$
H_{\nu}^{s}(\|z\|)=H_{\nu}(\|z\| / s)=f_{\nu}\left(\|z\|^{2} / s^{2}\right) .
$$

For these new Hamiltonians the level sets consisting of 1-periodic orbits are defined by the equations

$$
f_{\nu}^{\prime}\left(\|z\|^{2} / s^{2}\right)=s^{2} k \pi
$$

When $k$ is any integer in $\left[1,2^{\nu+3} / \pi s^{2}\right)$ each of these equations has two solutions, $\|z\|^{2}=s^{2} t_{\nu, k}^{ \pm}(s)$, where $t_{\nu, k}^{-}(s)$ is in $\left[1-1 / 2^{\nu}, 1-3 / 2^{\nu+2}\right)$ and $t_{\nu, k}^{+}(s)$ is in $\left(1-1 / 2^{\nu+1}, 1-1 / 2^{\nu+2}\right]$. We also have $t_{\nu, k}^{ \pm}(s) \rightarrow t_{\nu, k}^{ \pm}$as $s \rightarrow 1$ for all $k$ and $\nu$.

The actions of the orbits on these level sets are equal to

$$
\mathcal{A}_{\nu, k}^{ \pm}(s)=f_{\nu}\left(t_{\nu, k}^{ \pm}(s)\right)-t_{\nu, k}^{ \pm}(s) \pi k s^{2} .
$$

As $\nu \rightarrow \infty$, the actions $\mathcal{A}_{\nu, k}^{-}(s)$ decrease exponentially to negative infinity, and the actions $\mathcal{A}_{\nu, k}^{+}(s)$ decrease monotonically to $-\pi k s^{2}$.

Just as above, $H_{\nu}^{s}$ is in $\mathcal{H}^{a, b}$ for sufficiently large $\nu$, and the only orbits with action in $[a, b)$ are those on the level sets $\|z\|^{2}=s^{2} t_{\nu, k}^{+}(s)$ for $k \in\left[1,-a / \pi s^{2}\right)$. The same arguments also show that the functions $H_{\nu}^{s}$ (eventually) form an upward exhausting sequence for $\mathrm{SH}^{[a, b)}\left(B_{s}\right)$. In particular, this is true for $s=R$.

\subsubsection{Proof of Claim 4.5}

By the scaling properties of the functions $H_{\nu}^{s}$ it suffices to prove Claim 4.5 for the case $s=1$. Recall that the periodic orbits of $H_{\nu}$ which lie on the level sets defined by $\|z\|^{2}=t_{\nu, k}^{-}$have actions which decrease exponentially as $\nu \rightarrow \infty$. Hence, when considering bounded intervals of actions and large enough $\nu$, the only relevant periodic orbits are those on the level sets of $H_{\nu}$ defined by $\|z\|^{2}=t_{\nu, k}^{+}$. The actions of these orbits are given by

$$
\mathcal{A}_{\nu, k}^{+}(1)=f_{\nu}\left(t_{\nu, k}^{+}\right)-t_{\nu, k}^{+} \pi k
$$

which decreases to $-\pi k$ monotonically. The following result is now obvious. 
Lemma 4.10. Let $l$ be a non-negative integer and let $-(l+1) \pi<\alpha \leq \beta \leq-l \pi$. Then for sufficiently large $\nu$

$$
\operatorname{HF}^{[\alpha, \beta)}\left(H_{\nu}\right)=0 .
$$

Now, consider intervals of the form $[-k \pi-\epsilon,-k \pi+\delta)$ where $0<\epsilon, \delta<\pi$ and $k \in \mathbb{Z}^{+}$. By the same reasoning as above, for sufficiently large $\nu$, the only level set with action in this interval is $\|z\|^{2}=t_{\nu, k}^{+}$.

Lemma 4.11. For any $0<\epsilon, \delta<\pi$ and all $\nu$ sufficiently large

$$
\mathrm{HF}_{*}^{[-k \pi-\epsilon,-k \pi+\delta)}\left(H_{\nu}\right) \cong H_{*+j(k)}\left(S M, \mathbb{Z}_{2}\right),
$$

where $S M$ is the sphere bundle of the normal bundle to $M$ in $W$ and

$$
j(k)=\frac{1}{2} \operatorname{dim} W+k \operatorname{codim} M-1 .
$$

Proof. The assertion, up to the value of the shift $j(k)$, follows immediately from Theorem 3.5 provided that the set of periodic orbits in $\|z\|^{2}=t_{\nu, k}^{+}$is MorseBott. However, it is straightforward to check that the Morse-Bott condition is equivalent, for the Hamiltonians in questions, to the condition $f_{\nu}^{\prime \prime}\left(t_{k}^{+}\right) \neq 0$, [BPS, Lemma 5.3.2].

The value of $j(k)$ is determined by considering an explicit perturbation of the Hamiltonian $H_{\nu}$. Any small perturbation $\tilde{H}_{\nu}$ of $H_{\nu}$ is still in $\mathcal{H}^{-k \pi-\epsilon,-k \pi+\delta}$ and satisfies

$$
H F_{*}^{[-k \pi-\epsilon,-k \pi+\delta)}\left(\tilde{H}_{\nu}\right) \cong \mathrm{HF}_{*}^{[-k \pi-\epsilon,-k \pi+\delta)}\left(H_{\nu}\right) .
$$

It is possible to describe an explicit perturbation whose periodic orbits with actions in the interval $(-k \pi-\epsilon,-k \pi+\delta)$ are all nondegenerate and have Conley-Zehnder indices in the range

$$
[-(2 k+1) n-m+1,-(2 k-1) n+m],
$$

where $2 m=\operatorname{dim} M$ and $2 n=\operatorname{codim} M$.

Since this range has length $2(n+m)-1=\operatorname{dim}(S M)$ and these periodic orbits must generate the restricted Floer homology, it follows from the isomorphism in Theorem 3.5 that the shift in grading must be

$$
j(k)=(2 k+1) n+m-1=\frac{1}{2} \operatorname{dim} W+k \operatorname{codim} M-1 .
$$

For the sake of completeness we now describe the explicit perturbation mentioned above. First consider the case when $M$ is the origin in $W=\mathbb{C}^{n}$. Then the functions $H_{\nu}$ are smooth radial functions and we can perturb $H_{\nu}$ in the manner described in detail in [FHW, Section 2.1]. This procedure can be be summarized as follows. One first approximates $H_{\nu}(z)=f_{\nu}\left(\|z\|^{2}\right)$ by $f_{\nu}(q(z))$, where $q(z)$ is a quadratic form which has eigenvalues that are close to one and are independent over the rationals. With this, the 1-periodic level set $\|z\|^{2}=t_{\nu, k}^{+}$gives rise to $n$ 
isolated periodic orbits. One then makes additional time-dependent perturbations which are supported near these isolated orbits and which split each of them into exactly two nondegenerate orbits. The overall perturbation of $H_{\nu}$ then has exactly $2 n$ periodic orbits with actions in the interval $(-k \pi-\epsilon,-k \pi+\delta)$. Their Conley-Zehnder indices are the integers in the range

$$
[-(2 k+1) n+1,-(2 k-1) n] .
$$

(The difference between these index values and those in $[\mathrm{FHW}]$ is due to the fact that the Hamiltonians $H_{\nu}$ are concave near the level sets $\|z\|^{2}=t_{\nu, k}^{+}$, whereas the Hamiltonians considered in $[\mathrm{FHW}]$ are convex. The calculation of the indices is straightforward but rather tedious, and involves only the definition and standard properties of the Conley-Zehnder index. We refer the reader to, e.g., [Sa], for a description of the Conley-Zehnder index and further references.)

To deal with the general case of a tubular neighborhood of $M \subset W$, we first consider a perturbation of $H_{\nu}$ of the form $f_{\nu}\left(\|z\|^{2}\right)+g(p)$. Here $g$ is the pull back to $E$ of a $C^{2}$-small Morse function on $M$. Under this perturbation, the 1-periodic level set $\|z\|^{2}=t_{\nu, k}^{+}$gives rise to a sphere of 1-periodic orbits in each of the fibres of $E$ over the critical points of $g$. More precisely, in the fibre over a critical point $p_{0} \in M$, the flow of the perturbed function agrees with model described above for $\mathbb{C}^{n}$ and the Hamiltonian $f_{\nu}\left(\|z\|^{2}\right)+g\left(p_{0}\right)$. The sphere of 1-periodic orbits in the fibre over $p_{0}$ is close to the original sphere of 1-periodic orbits for $H_{\nu}$ and can be perturbed as in the case of $\mathbb{C}^{n}$ above. This results in exactly $2 n$ nondegenerate periodic orbits with actions in the interval $(-k \pi-\epsilon,-k \pi+\delta)$. The contribution of $g$ to the Hamiltonian flow shifts their Conley-Zehnder indices which now cover the range

$$
\left[-(2 k+1) n+1+\mu_{M}\left(p_{0}\right)-m,-(2 k-1) n+\mu_{M}\left(p_{0}\right)-m\right],
$$

where $\mu_{M}\left(p_{0}\right)$ denotes the Morse index of $p_{0}$. We perturb each fibre over the critical points of $g$ in the same way. The periodic orbits of the final perturbation with action in $(-k \pi-\epsilon,-k \pi+\delta)$ are all nondegenerate and have Conley-Zehnder indices in the interval

$$
[-(2 k+1) n-m+1,-(2 k-1) n+m]
$$

as required.

Proceeding with the proof of Claim 4.5, we first show that for any constant $c<a$, where $c$ is not a negative integer multiple of $\pi$, the map

$$
\Pi: \mathrm{HF}_{n_{0}}^{[c, b)}\left(H_{\nu}\right) \rightarrow \mathrm{HF}_{n_{0}}^{[a, b)}\left(H_{\nu}\right)
$$

is an isomorphism provided that $\nu$ is large enough.

To do this, we consider the following exact sequence which is a part of the exact triangle $\triangle_{c, a, b}\left(H_{\nu}\right)$ (see Section 3.2.4.):

$$
\mathrm{HF}_{n_{0}}^{[c, a)}\left(H_{\nu}\right) \rightarrow \mathrm{HF}_{n_{0}}^{[c, b)}\left(H_{\nu}\right) \stackrel{\Pi}{\rightarrow} \operatorname{HF}_{n_{0}}^{[a, b)}\left(H_{\nu}\right) \rightarrow \mathrm{HF}_{n_{0}-1}^{[c, a)}\left(H_{\nu}\right) .
$$


First, assume that $c$ and $a$ are contained in an interval of the form $(-\pi(k+$ $1),-\pi k]$. Then Lemma 4.10 immediately implies that the first and last terms in (8) vanish for sufficiently large $\nu$, and hence $\Pi$ is an isomorphism.

Next, we look at the case where $c$ and $a$ are in adjacent intervals, i.e.,

$$
-\pi(k+1)<c<-\pi k<a \leq-\pi(k-1)
$$

for some integer $k \geq 2$ (since $a<-\pi$ ). By Lemma 4.11, if $\nu$ is large enough,

$$
\mathrm{HF}_{n_{0}}^{[c, a)}\left(H_{\nu}\right)=H_{n_{0}+j(k)}\left(S M, \mathbb{Z}_{2}\right)=0,
$$

because $n_{0}+j(1)=\operatorname{dim} S M$ and $j(1)<j(k)$. (The sequence $j(k)$ is increasing.) In a similar manner, we obtain

$$
\mathrm{HF}_{n_{0}-1}^{[c, a)}\left(H_{\nu}\right)=H_{n_{0}+j(k)-1}\left(S M, \mathbb{Z}_{2}\right)=0,
$$

when $k \geq 2$. Hence, in this case, $\Pi$ is again an isomorphism.

Now, for any $c<a$ such that $c \notin \pi \mathbb{Z}^{+}$, the map $\Pi$ can be factored as a composition of isomorphisms for points in adjacent intervals. More precisely, $\Pi=$ $\Pi_{l} \circ \Pi_{l-1} \circ \cdots \circ \Pi_{1}$, where

$$
\Pi_{j}: \mathrm{HF}_{n_{0}}^{\left[c_{j}, b\right)}\left(H_{\nu}\right) \rightarrow \mathrm{HF}_{n_{0}}^{\left[c_{j-1}, b\right)}\left(H_{\nu}\right)
$$

The constants $c_{j}$ are chosen to be in adjacent intervals so that $c_{0}=a$ and $c_{j} \notin-\pi \mathbb{Z}^{+}$for $j \geq 1$. The previous argument then implies that each $\Pi_{j}$ is an isomorphism for sufficiently large $\nu$. Thus, the same is true for $\Pi$.

It remains to show that $\operatorname{HF}_{n_{0}}^{[c, b)}\left(H_{\nu}\right)=\operatorname{HF}_{n_{0}}^{[a, b)}\left(H_{\nu}\right)=\mathbb{Z}_{2}$. The above argument reduces the problem to the calculation of $\operatorname{HF}_{n_{0}}^{[\alpha, b)}\left(H_{\nu}\right)$ for $-2 \pi<\alpha<-\pi$ and $\nu$ large enough. By Lemma 4.11, we have

$$
\operatorname{HF}_{n_{0}}^{[\alpha, b)}\left(H_{\nu}\right)=H_{n_{0}+j(1)}\left(S M, \mathbb{Z}_{2}\right)=H_{\operatorname{dim} S M}\left(S M, \mathbb{Z}_{2}\right)=\mathbb{Z}_{2}
$$

since $n_{0}+j(1)=\operatorname{dim} S M$. This completes the proof of Claim 4.5.

\subsubsection{Proof of Claim 4.6}

Again by the scaling properties of the functions $H_{\nu}^{s}$, it suffices to prove Claim 4.6 when $s_{0}=1$ and $1<s_{1}<\sqrt{1+1 /\left(k^{\prime}+1\right)}$.

The proof is divided into two parts. First we prove that there is a constant $c<a$ such that the homomorphism

$$
\hat{\sigma}_{H_{\nu}^{s_{1}} H_{\nu}}: \mathrm{HF}^{[a, b)}\left(H_{\nu}\right) \rightarrow \mathrm{HF}^{[c, b)}\left(H_{\nu}^{s_{1}}\right)
$$

is well defined for sufficiently large $\nu$. Then we use Lemma 3.8 to prove that this map is an isomorphism.

For any $c<a$, the functions $H_{\nu}^{s_{1}}$ are in $\mathcal{H}^{c, b}$ for all sufficiently large $\nu$. This follows again from the fact that, as $\nu$ increases, the actions $\mathcal{A}_{\nu, k}^{+}\left(s_{1}\right)$ decrease monotonically to $-\pi k s_{1}^{2}$ and the actions $\mathcal{A}_{\nu, k}^{-}\left(s_{1}\right)$ decrease exponentially. Hence, 
to show that the map $\hat{\sigma}_{H_{\nu}^{s_{1}} H_{\nu}}$ in (9) is well defined, it remains to find a constant $c<a$ for which the Floer chain map $\sigma_{H_{\nu}^{s_{1}} H_{\nu}}$ satisfies

$$
\sigma_{H_{\nu}^{s_{1}} H_{\nu}}\left(\mathrm{CF}^{a}\left(H_{\nu}\right)\right) \subset \mathrm{CF}^{c}\left(H_{\nu}^{s_{1}}\right)
$$

for all sufficiently large $\nu$.

To prove this, we must approximate the functions $H_{\nu}$ and $H_{\nu}^{s_{1}}$ by functions $\tilde{H}_{\nu}$ and $\tilde{H}_{\nu}^{s_{1}}$, respectively, which have the nondegeneracy property $\left(*^{b}\right)$. Then we must find a constant $c<a$ such that

$$
\sigma_{\tilde{H}_{\nu}^{s_{1}} \tilde{H}_{\nu}}\left(\mathrm{CF}^{a}\left(\tilde{H}_{\nu}\right)\right) \subset \mathrm{CF}^{c}\left(\tilde{H}_{\nu}^{s_{1}}\right)
$$

for sufficiently large $\nu$ (see Section 3.1.3.). In other words, for every $\tilde{x} \in \mathcal{P}^{a}\left(\tilde{H}_{\nu}\right)$ we must show that its image $\sigma_{\tilde{H}_{\nu}^{s_{1}} \tilde{H}_{\nu}}(\tilde{x})$ only contains elements $\tilde{y} \in \mathcal{P}\left(\tilde{H}_{\nu}^{s_{1}}\right)$ which satisfy $\mathcal{A}_{\tilde{H}_{\nu}^{s_{1}}}(\tilde{y})<c$.

Let $\tilde{y}$ be in $\sigma_{\tilde{H}_{\nu}^{s_{1}} \tilde{H}_{\nu}}(\tilde{x})$. The fact that the chain map $\sigma_{\tilde{H}_{\nu}^{s_{1}} \tilde{H}_{\nu}}$ is defined using a monotone homotopy already implies that

$$
\mathcal{A}_{\tilde{H}_{\nu}^{s_{1}}}(\tilde{y}) \leq \mathcal{A}_{\tilde{H}_{\nu}}(\tilde{x})<a .
$$

We will show that our choice of $s_{1}$ forces the action to drop by some fixed value $a-c>0$ whenever $\nu$ is large enough and the approximations $\tilde{H}_{\nu}$ and $\tilde{H}_{\nu}^{s_{1}}$ are close enough to the original Hamiltonians.

Our choice of $k^{\prime}$ implies that $a \in\left[-\left(k^{\prime}+1\right) \pi,-k^{\prime} \pi\right)$, and our choice of $s_{1}$ yields

$$
-k^{\prime} \pi s_{1}^{2}>-\left(k^{\prime}+1\right) \pi>-\left(k^{\prime}+1\right) \pi s_{1}^{2}>-\left(k^{\prime}+2\right) \pi>-\left(k^{\prime}+2\right) \pi s_{1}^{2} .
$$

We will prove that $\mathcal{A}_{\tilde{H}_{\nu}^{s_{1}}}(\tilde{y})<c$ for some $c<a$ when $a \neq-\left(k^{\prime}+1\right) \pi$. To do this we will utilize the first two inequalities from (12). The case $a=-\left(k^{\prime}+1\right) \pi$ can be dealt with in an entirely similar manner using the last two inequalities from (12).

We recall that, as $\nu \rightarrow \infty$, the action values $\mathcal{A}_{\nu, k}^{+}(s)$ decrease monotonically to $-k \pi s^{2}$ and the action values $\mathcal{A}_{\nu, k}^{-}(s)$ decrease exponentially. When $s=1$ and $a \neq-\left(k^{\prime}+1\right) \pi$, this implies that for any $\epsilon>0$ we can choose $\nu$ to be sufficiently large so that every $x \in \mathcal{P}^{a}\left(H_{\nu}\right)$ satisfies

$$
\mathcal{A}_{H_{\nu}}(x)<-\left(k^{\prime}+1\right) \pi+\epsilon .
$$

By Lemma 3.1, the 1-periodic orbits of the approximations $\tilde{H}_{\nu}$ can be assumed to have actions arbitrarily close to $\mathcal{S}\left(H_{\nu}\right)$. Hence, we also have

$$
\mathcal{A}_{\tilde{H}_{\nu}}(\tilde{x})<-\left(k^{\prime}+1\right) \pi+\epsilon,
$$

for every $\tilde{x} \in \mathcal{P}^{a}\left(\tilde{H}_{\nu}\right)$

Let $N$ be any positive integer and let $\delta>0$. As above, the asymptotic behavior of the actions $\mathcal{A}_{\nu, k}^{ \pm}(s)$, for $s=s_{1}$, implies that, for $\nu$ sufficiently large and $\tilde{H}_{\nu}^{s_{1}}$ sufficiently close to $H_{\nu}^{s_{1}}$, the following alternative holds for every $\tilde{y} \in \mathcal{P}^{0}\left(\tilde{H}_{\nu}^{s_{1}}\right)$ :

Either

$$
-k \pi s_{1}^{2}<\mathcal{A}_{\tilde{H}_{\nu}^{s_{1}}}(\tilde{y})<-k \pi s_{1}^{2}+\delta \text { for some } k=1,2, \ldots, N
$$




$$
\mathcal{A}_{\tilde{H}_{\nu}^{s_{1}}}(\tilde{y})<-N \pi s_{1}^{2} .
$$

We now fix the constant $c$ so that

$$
c \in\left(-\left(k^{\prime}+1\right) \pi s_{1}^{2}, a\right) .
$$

We also choose $\nu$ and the approximations $\tilde{H}_{\nu}$ and $\tilde{H}_{\nu}^{s_{1}}$ so that the constants in (13), (14), and (15) satisfy the following bounds:

$$
\epsilon<-k^{\prime} \pi s_{1}^{2}+\left(k^{\prime}+1\right) \pi, \quad \delta<c+\left(k^{\prime}+1\right) \pi s_{1}^{2}, \quad \text { and } \quad N>k^{\prime}+2 .
$$

The inequalities (11) and (13), together with our upper bound for $\epsilon$, imply that

$$
\mathcal{A}_{\tilde{H}_{\nu}^{s_{1}}}(\tilde{y})<-k^{\prime} \pi s_{1}^{2} .
$$

It now follows, from the alternative described in (14) and (15), and our lower bound for $N$, that

$$
\mathcal{A}_{\tilde{H}_{\nu}^{s_{1}}}(\tilde{y})<-\left(k^{\prime}+1\right) \pi s_{1}^{2}+\delta .
$$

Finally, our upper bound for $\delta$ implies that

$$
\mathcal{A}_{\tilde{H}_{\nu}^{s_{1}}}(\tilde{y})<c .
$$

When $a=-\left(k^{\prime}+1\right) \pi$, the constant $c$ can be chosen to be in the interval $\left(-\left(k^{\prime}+2\right) \pi s_{1}^{2}, a\right)$.

Now that we have shown that the map $\hat{\sigma}_{H_{\nu}^{s_{1}} H_{\nu}}: \operatorname{HF}^{[a, b)}\left(H_{\nu}\right) \rightarrow \operatorname{HF}^{[c, b)}\left(H_{\nu}^{s_{1}}\right)$ is well defined, it remains to show that it is an isomorphism for large $\nu$. Consider the monotone homotopy $H_{\nu}^{\phi(s)}$ from $H_{\nu}$ to $H_{\nu}^{s_{1}}$, where $\phi: \mathbb{R} \rightarrow\left[1, s_{1}\right]$ is a smooth increasing function which equals 1 for $s \leq-1$ and equals $s_{1}$ for $s \geq 1$. As above, we denote the actions of the non-constant contractible 1-periodic orbits of the function $H_{\nu}^{\phi(s)}$ by $\mathcal{A}_{\nu, k^{\prime}+1}^{ \pm}(\phi(s))$. Again, as $\nu$ goes to infinity, the actions $\mathcal{A}_{\nu, k}^{+}(\phi(s))$ decrease monotonically to $-k \pi(\phi(s))^{2}$ and the actions $\mathcal{A}_{\nu, k}^{-}(\phi(s))$ decrease to negative infinity.

For the case $a \neq-\left(k^{\prime}+1\right) \pi$, we fix $c \in\left(-\left(k^{\prime}+1\right) \pi s_{1}^{2}, a\right)$ so that $\hat{\sigma}_{H_{\nu}^{s_{1}} H_{\nu}}$ is well defined. Let $a_{s}$ be a continuous family of numbers, for $s \in[-1,1]$, such that $a_{-1}=a, a_{1}=c$, and

$$
\mathcal{A}_{\nu, k^{\prime}+1}^{+}(\phi(s))<a_{s}<\mathcal{A}_{\nu, k^{\prime}}^{+}(\phi(s))
$$

for all $s \in[-1,1]$. For large $\nu$, it is clear from their asymptotic behavior that none of the actions $\mathcal{A}_{\nu, k}^{ \pm}(\phi(s))$ is ever equal to $a_{s}$. In other words, if one considers the graphs of the actions $\mathcal{A}_{\nu, k}^{ \pm}(\phi(s))$ over the interval $[-1,1]$, they do not intersect the graph of the family $a_{s}$ because they are either too close to the graphs of the functions $-k \pi s$ or their values are too negative. It follows that the $H_{\nu}^{\phi(s)}$ are in $\mathcal{H}^{a_{s}, b}$ for all $s \in[-1,1]$ and Lemma 3.8 then implies that $\hat{\sigma}_{H_{\nu}^{s_{1}} H_{\nu}}$ is an isomorphism.

A similar argument applies in the case $a=-\left(k^{\prime}+1\right) \pi$. This completes the proof of Claim 4.6 and hence Proposition 4.2 . 


\section{Homological symplectic capacity}

Closely following [FHW], we will now define a certain relative homological capacity. The results of this paper imply the finiteness of this capacity in various cases.

As above, let $W$ be a geometrically bounded symplectically aspherical manifold. For an open subset $U \subset W$, we set

$$
\mathrm{SH}^{[a, 0)}(U)=\underset{b \nearrow 0}{\lim _{b \nearrow}} \mathrm{SH}^{[a, b)}(U) .
$$

Furthermore, for a compact subset $Z \subset W$, we define its symplectic homology as

$$
\mathrm{SH}^{[a, 0)}(Z)=\lim _{U \searrow Z} \mathrm{SH}^{[a, 0)}(U)
$$

where the limit is taken over all open sets $U \supset Z$. By the definition of the inverse limit, we have the projection map

$$
\phi_{U}^{a}: \mathrm{SH}^{[a, 0)}(Z) \rightarrow \mathrm{SH}^{[a, 0)}(U)
$$

for every open set $U \supset Z$.

Example 5.1. Let $M$ be a closed symplectic submanifold of $W$ and let $B_{R}$ be the tubular neighborhoods of $M$ constructed in Section 4.1. As readily follows from Proposition 4.2, $\mathrm{SH}_{n_{0}}^{[a, 0)}(M)=\mathbb{Z}_{2}$, where $n_{0}=\frac{3}{2} \operatorname{dim} W$-codim $M-1$. Moreover, $\phi_{B_{R}}^{a}: \mathrm{SH}_{n_{0}}^{[a, 0)}(M) \rightarrow \mathrm{SH}_{n_{0}}^{[a, 0)}\left(B_{R}\right)$ is an isomorphism, provided that $a<-\pi R^{2}$. Furthermore, $\mathrm{SH}^{[a, 0)}\left(B_{R}\right)=0$ if $-\pi R^{2}<a<0$.

Define the relative homological capacity of $(U, Z)$ as

$$
\mathrm{c}_{\mathrm{SH}}(U, Z)=\inf \left\{-a \mid \phi_{U}^{c} \neq 0 \text { for all } c<a\right\} .
$$

Clearly, $c_{\mathrm{SH}}$ is an invariant of symplectomorphisms of the ambient manifold $W$. The fact that $c_{\mathrm{SH}}$ is a symplectic capacity (defined only on submanifolds of $W$ ) can be easily verified using Proposition 4.2. In other words, we have

\section{Theorem 5.2.}

(1) [Invariance]. The relative capacity $\mathrm{c}_{\mathrm{SH}}$ is an invariant of symplectomorphisms of $W$.

(2) [Monotonicity]. Let $Z^{\prime} \subset Z \subset U \subset U^{\prime}$. Then $\mathrm{c}_{\mathrm{SH}}(U, Z) \leq \mathrm{c}_{\mathrm{SH}}\left(U^{\prime}, Z\right)$ and $\mathrm{c}_{\mathrm{SH}}(U, Z) \leq \mathrm{c}_{\mathrm{SH}}\left(U, Z^{\prime}\right)$.

(3) [Homogeneity]. For any constant $a>0$,

$$
c_{\mathrm{SH}}(U, Z, a \omega)=a \mathrm{c}_{\mathrm{SH}}(U, Z, \omega) .
$$

(4) [Normalization]. Assume that $M$ is a closed symplectic submanifold of a geometrically bounded symplectically aspherical manifold $W$ and let $B_{R}$ be a symplectic tubular neighborhood of $M$ in $W$ of radius $R>0$ (see Section 4.1). Then $\mathrm{c}_{\mathrm{SH}}\left(B_{R}, M\right)=\pi R^{2}$. 
Here the first three assertions are obvious. To prove the last assertion, note that $\mathrm{c}_{\mathrm{SH}}\left(B_{R}, M\right) \leq \pi R^{2}$ by Proposition 4.2. On the other hand, $\mathrm{SH}^{[a, 0)}\left(B_{R}\right)=0$ when $-\pi R^{2}<a<0$ as readily follows from the analysis carried out in Section 4.2.3. (see Lemma 4.10). Thus, $\mathrm{c}_{\mathrm{SH}}\left(B_{R}, M\right) \geq \pi R^{2}$, which completes the proof of the last assertion.

The capacity $\mathrm{c}_{\mathrm{SH}}(U$, point $)$ is essentially the homological capacity introduced in [FHW]. Once, $\mathrm{c}_{\mathrm{SH}}(U, Z)<\infty$, the symplectic homology of $V$ is non-zero for any open subset $V$ of $U$ such that $Z \subset V \subset U$. Then a suitable version of Proposition 3.14 implies the nearby existence theorem in $(U, Z)$, i.e., the existence of contractible periodic orbits on a dense of levels of any function attaining an isolated minimum on $Z$. Yet, the finiteness of this capacity does not seem to imply the existence of periodic orbits for almost all energy levels as does the HoferZehnder capacity. A different version of a relative capacity, a relative analogue of the Hofer-Zehnder capacity, which is sufficient for proving a version of the almost existence theorem is introduced and analyzed in detail in [GG].

Finally, we note that the relative homological capacity $\mathrm{c}_{\mathrm{SH}}$ is different from, although apparently related to, the one introduced in [BPS].

\section{References}

[AL] M. Audin and J. Lafontaine (Eds), Holomorphic curves in symplectic geometry, Progress in Mathematics, 117, Birkhäuser Verlag, Basel, 1994.

[Bi] P. Biran, Lagrangian barriers and symplectic embeddings, Geom. Funct. Anal. 11 (2001), 407-464.

[BPS] P. Biran, L. Polterovich and D. Salamon, Propagation in Hamiltonian dynamics and relative symplectic homology, Duke Math. J. 119 (2003), 65-118.

[CFH] K. Cieliebak, A. Floer and H. Hofer, Symplectic homology, II. A general construction, Math. Z. 218 (1995), 103-122.

[CFHW] K. Cieliebak, A. Floer, H. Hofer and K. Wysocki, Applications of symplectic homology, II. Stability of the action spectrum, Math. Z. 223 (1996), 27-45.

[EG] Y. Eliashberg and M. Gromov, Convex symplectic manifolds, in Several complex variables and complex geometry, Part 2 (Santa Cruz, CA, 1989), 135-162, Proc. Sympos. Pure Math., 52, Part 2, Amer. Math. Soc., Providence, RI, 1991.

[F11] A. Floer, Morse theory for Lagrangian intersections, J. Diff. Geom. 28 (1988), 513-547.

[Fl2] A. Floer, Witten's complex and infinite dimensional Morse Theory, J. Diff. Geom. 30 (1989), 202-221.

[Fl3] A. Floer, Symplectic fixed points and holomorphic spheres, Commun. Math. Phys. 120 (1989), 575-611.

[FH] A. Floer and H. Hofer, Symplectic homology, I. Open sets in $\mathbb{C}^{n}$, Math. Z. 215 (1994), $37-88$.

[FHW] A. Floer, H. Hofer and K. Wysocki, Applications of symplectic homology, I. Math. Z. 217 (1994), 577-606.

[Gi] V. L. Ginzburg, On closed trajectories of a charge in a magnetic field. An application of symplectic geometry, in: Contact and symplectic geometry (Cambridge, 1994), 131-148, Publ. Newton Inst., 8, Cambridge Univ. Press, Cambridge, 1996.

[GG] V. L. Ginzburg and B. Z. Gürel, Relative Hofer-Zehnder capacity and periodic orbits in twisted cotangent bundles, Preprint 2003, math.DG/0301073; to appear in Duke Math. J. 
[GK1] V. L. Ginzburg and E. Kerman, Periodic orbits in magnetic fields in dimensions greater than two, in: Geometry and topology in dynamics (Winston-Salem, NC, 1998/San Antonio, TX, 1999), 113-121, Contemp. Math., 246, Amer. Math. Soc., Providence, RI, 1999.

[GK2] V. L. Ginzburg and E. Kerman, Periodic orbits of Hamiltonian flows near symplectic extrema, Pacific Journal of Mathematics 206 (2002), 69-91.

[Hi] M. Hirsch, Differential Topology, Springer-Verlag, Graduate Texts in Mathematics; New York-Heidelberg-Berlin, 1976.

[HZ] H. Hofer and E. Zehnder, Symplectic invariants and Hamiltonian dynamics, Birkhäuser, Advanced Texts; Basel-Boston-Berlin, 1994

[Ji] M. Y. Jiang, Periodic solutions of Hamiltonian systems on hypersurfaces in a torus, Manuscripta Math. 85 (1994), 307-321.

[Ke] E. Kerman, Periodic orbits of Hamiltonian flows near symplectic critical submanifolds, $\operatorname{IMRN}$ (1999), no. 17, 954-969.

[Lu] G. Lu, The Weinstein conjecture on some symplectic manifolds containing the holomorphic spheres, Kyushu J. Math. 52 (1998), 331-351.

[Mac] L. Macarini, Hofer-Zehnder capacity and Hamiltonian circle actions, Preprint 2002, math.SG/0205030.

[Pol] L. Polterovich, Geometry on the group of Hamiltonian diffeomorphisms, in: Proceedings of the International Congress of Mathematicians, Vol. II (Berlin, 1998). Doc. Math. 1998, Extra Vol. II, 401-410 (electronic).

[Po] M. Poźniak, Floer homology, Novikov rings and clean intersections, in Northern California Symplectic Geometry Seminar, 119-181, Amer. Math. Soc. Transl. Ser. 2, 196, Amer. Math. Soc., Providence, RI, 1999.

[Sa] D. A. Salamon, Lectures on Floer homology, in Symplectic Geometry and Topology, Eds: Y. Eliashberg and L. Traynor, IAS/Park City Mathematics series, 7, 1999, pp. $143-230$.

[Sc] M. Schwarz, On the action spectrum for closed symplectically aspherical manifolds, Pacific J. Math. 193 (2000), 419-461.

[Vi] C. Viterbo, Functors and computations in Floer homology with applications, I, Geom. Funct. Anal. 9 (1999), 985-1033.

Kai Cieliebak

Mathematisches Institut

Ludwig-Maximilians-Universität

Theresienstr. 39

80333 München

Germany

e-mail: kai@mathematik.uni-muenchen.de

Ely Kerman

Department of Mathematics

SUNY at Stony Brook

Stony Brook

NY 11794-3651

USA

e-mail: ely@math.sunysb.edu

(Received: January 14, 2003)
Viktor L. Ginzburg

Department of Mathematics

UC Santa Cruz

Santa Cruz

CA 95064

USA

e-mail: ginzburg@math.ucsc.edu 